\title{
Suppressive effects of Aspergillus fumigatus culture filtrates on human alveolar macrophages and polymorphonuclear leucocytes
}

\author{
T. Murayama, R. Amitani, Y. Ikegami, R. Nawada, W.J. Lee, F. Kuze
}

Suppressive effects of Aspergillus fumigatus culture filtrates on human alveolar macrophages and polymorphonuclear leucocytes. T. Murayama, R. Amitani, Y. Ikegami, R. Nawada, W.J. Lee, F. Kuze. @eERS Journals Ltd 1996.

ABSTRACT: Aspergillus spp., especially A. fumigatus (Af) can colonize the airways and the lungs with localized underlying conditions and further invade the surrounding lung tissues, even in subjects without systemic predisposing factors, presumably by escaping the local host defences.

To clarify the mechanisms of colonization and invasion of $A f$, we investigated the in vitro effects of $A f$ culture filtrates (ACFs) on the functions of human alveolar macrophages (AMs), and polymorphonuclear leucocytes (PMNs). ACFs were obtained by culturing clinically isolated $A f$ in Medium-199 at $37^{\circ} \mathrm{C}$ for 5 days.

In the study of phagocytosis of $A f$ conidia by human AMs, $52 \%$ of AMs ingested conidia during a $60 \mathrm{~min}$ incubation period in Medium-199. However, the percentage decreased to $24 \%$ when incubated with a final concentration of $30 \%$ ACF in Medium-199. With respect to the antichemotactic activity on human PMNs, $3 \%$ ACF was sufficient for significant suppression, and 30\% ACF completely inhibited the migration of PMNs. In addition, phorbol myristate acetate (PMA)-induced $\mathrm{O}_{2}^{-}$ release from PMNs was significantly suppressed in Medium-199 which included 12.5\% ACF or more. The antichemotactic activity of ACF was partially abolished by trypsin or chicken egg ovomacroglobulin. When ACF was separated into two fractions (molecular weight $>\mathbf{1 0}$ and $<\mathbf{1 0} \mathrm{kDa}$ ) by dialysis and centrifugation through CL-LGC filters, both fractions retained the antichemotactic activity.

We conclude that $A f$ produce several antiphagocytic factors, which can be responsible for the colonization of $A f$ in the bronchopulmonary tissues and allow this species to invade surrounding lung tissues in pulmonary aspergillosis by suppressing local host defences.

Eur Respir J., 1996, 9, 293-300.
Dept of Infection and Inflammation, Chest Disease Research Institute, Kyoto University, Kyoto, Japan.

Correspondence: T. Murayama Dept of Infection and Inflammation Chest Disease Research Institute Kyoto University

Sakyo-ku

Kyoto 606

Japan

Keywords: Alveolar macrophage antiphagocytic activity Aspergillus fumigatus

human phagocytes

polymorphonuclear leucocyte

Received: May 21995

Accepted after revision November 21995
Aspergillus fumigatus (Af), the commonest pathogenic Aspergillus species in humans, is known to cause a wide spectrum of illness ranging from saprophytic colonization of the bronchial trees to invasive and disseminated disease [1]. Although an acute progressive process occurs most commonly in systemic immunocompromised hosts, Af frequently colonizes lung residual cavities or injured airways, such as those in healed tuberculosis, bronchiectasis or cystic fibrosis, and occasionally chronic invasion of the surrounding lung tissues occurs even in patients without apparently compromised systemic host defences [1]. In contrast, pathogenic fungi other than Aspergillus species rarely colonize and invade the bronchopulmonary tissues in similar conditions. However, the mechanisms of colonization and local invasion by $A f$ are still poorly understood.

SCHAFFNER and co-workers [2] demonstrated selective protection against conidia of Aspergillus by macrophages and against mycelium of Aspergillus by polymorphonuclear leucocytes (PMNs). They concluded that these lines of defence co-operate in the control and elimination of the fungi [2]. Since the respiratory tract is regarded as the main entrance to the human body for conidia, alveolar macrophages (AMs) seem to play a vital role in preventing germination of conidia in the airways and the lungs. AMs are considered to ingest and kill conidia by nonoxidative mechanisms [3]. PMNs exert the second line of host defence against Aspergillus by killing the mycelial form that has evaded attack by AMs and has germinated in the lung tissue $[4,5]$. As Aspergillus hyphae have a long, dichotomously branched, filamentous shape and are nonphagocytosable by a single cell, adhesion to the hyphae and release of the reactive oxygen intermediates and cationic peptides are considered to play the key roles in damage to the hyphal form by PMNs. The strong clinical association of severe neutropenia or dysfunction of PMNs with the high prevalence of invasive aspergillosis also suggests the importance of 
PMNs in host defences against the fungi [6]. Thus, it appears that AMs and PMNs form a dual defence system against Aspergillus.

Recently, there have been several reports that some secondary metabolites of $A f$, as well as culture filtrates or cell extracts of $A f$, suppress the host defence mechanisms in vitro [7-13]. It has been speculated that mycotoxins, such as gliotoxin $[10,11,13]$ and fumagillin, and extracellular proteases [14] produced by $A f$ suppress the local host defences, and these suppressive effects may contribute to making colonization easier and, furthermore, leading to a chronic infectious process by $A f$. However, most previous workers who have investigated interactions between secondary metabolites of $A f$ and phagocytes in vitro have used cells obtained from experimental animals, and not human phagocytes. Therefore, little is known about the virulence determinant of $A f$ in human pulmonary aspergillosis. Consequently, it is necessary to clarify the interaction between $A f$ and human phagocytes, especially AMs and PMNs, before any extrapolation can be made to human diseases.

In the present study, the antiphagocytic effects of $A f$ culture filtrates (ACF) on human AMs and PMNs were evaluated in vitro, and in this paper the $A f$-derived factors which might play important roles in suppressing human phagocytic functions are discussed.

\section{Materials and methods}

\section{Preparation of fungal culture filtrates}

A clinical isolate of $A f$, YN strain, was derived from the sputum of a patient with chronic necrotizing pulmonary aspergillosis. A strain of Candida albicans was also clinically isolated from a patient with oral candidiasis. After growth on slants of potato dextrose agar (PDA) (Difco Laboratories) for 5 days at $30^{\circ} \mathrm{C}$, conidia $\left(2 \times 10^{7}\right)$ of YN strain of $A f$ and spores $\left(2 \times 10^{7}\right)$ of C. albicans were cultured in flasks containing $300 \mathrm{~mL}$ of Medium-199 (Gibco RBL), without shaking, at $37^{\circ} \mathrm{C}$. After 5 days of culture, the fungi were removed by passing the medium through gauze, and each culture filtrate was sterilized by passage through Millex-GV $0.22 \mu \mathrm{m}$ Millipore-filters (Millipore Co.) and stored at $-80^{\circ} \mathrm{C}$. Before use in experiments, $\mathrm{pH}$ of the culture filtrates was adjusted to 7.4 with $1 \mathrm{M} \mathrm{HCl}$. The sterility of the culture filtrates was tested by 3 days of culture on PDA plates at $37^{\circ} \mathrm{C}$.

To assess the change in biological activities of the culture filtrates, a $5 \mathrm{~mL}$ aliquot of culture fluid was taken daily for 10 days from a flask containing $300 \mathrm{~mL}$ of Medium-199 and $2 \times 10^{7}$ conidia at the beginning of the culture, and stored at $-80^{\circ} \mathrm{C}$ after sterilization through Millex-GV filters. It was found that antichemotactic activity became apparent at 3 days and reached a peak at 5 days after the beginning of the culture, just as the protein concentration reached a plateau $\left(5 \mu \mathrm{g} \cdot \mathrm{mL}^{-1}\right)$. In this study, therefore, 5 day culture filtrates were used.

\section{Cell preparations}

Human AMs were isolated from the bronchoalveolar lavage (BAL) fluid of eight healthy adult volunteers who gave their informed consent. After centrifugation, the pellets were resuspended in Medium-199 containing $5 \%$ heat-inactivated $\mathrm{AB}$ serum. The cell suspensions were dispensed into Teflon tubes (Nalge Co.), which did not allow attachment to the tube wall [15]. More than $90 \%$ of the cell population was comprised of AMs. Fresh PMNs were prepared from heparinized venous blood obtained from an additional 10 healthy adult volunteers by sedimentation in $2 \%$ dextran, followed by centrifugation on a Ficoll gradient. Contaminating red blood corpuscles (RBCs) were lysed with hypotonic saline, and PMNs were washed and resuspended with Medium-199 in polypropylene tubes (Becton Dickinson Co.). Giemsa-stained cytospin preparations of the PMN suspensions typically showed more than 95\% PMNs. These procedures were approved by the Ethics Committee of our department. In each experiment, the viability of the cells, tested by trypan blue exclusion, was found to be greater than $95 \%$ in the medium alone and greater than $90 \%$ in the medium with culture filtrates for $2 \mathrm{~h}$.

\section{Ingestion assay}

Three hundred and fifty microlitres of Medium-199 containing floating AMs $\left(1 \times 10^{6}\right)$ and conidia of $A f$, YN strain $\left(5 \times 10^{6}\right)$ were mixed with $150 \mu \mathrm{L}$ of ACF or Medium-199. Each mixture was incubated in a waterbath, with shaking, at $37^{\circ} \mathrm{C}$ for $15-60 \mathrm{~min}$. Immediately after incubation, the mixtures were placed on ice to inhibit further phagocytosis and all tubes were washed twice with cold $0.1 \%$ ethylenediamine tetra-acetic acid (EDTA)-phosphate-buffered saline (PBS). Phagocytosis of conidia was assayed by the fluorescence quenching method [16]. Briefly, conidia prelabelled with fluorescein isothiocyanate (Sigma) were mixed with AMs. Immediately after incubation, each drop of suspension and trypan blue were placed together on a slide and examined under an ultra violet (UV) microscope (Nikon Fluophoto), and then the percentage of AMs ingesting conidia was calculated. Cells incubated with viable conidia were attached to the glass slides by the cytospin procedure and stained with May-Grünwald Giemsa. Five hundred AMs per slide were analysed for ingestion of conidia under light microscopy. The phagocytic index showed the average number of conidia ingested by 100 AMs.

Latex beads (Fluoresbrite carboxylate microspheres, $2.2 \mu \mathrm{m}$ in diameter; Polysciences Inc.) were added to the AMs $\left(2 \times 10^{6} \cdot \mathrm{mL}^{-1}\right)$ suspensions pretreated with culture filtrates (20 and $50 \%$ of $\mathrm{ACF}$, and $50 \%$ of C. albicans culture filtrates) at a ratio of 10:1 for beads:AMs, and then incubated for $30 \mathrm{~min}$ at $37^{\circ} \mathrm{C}$. The controls were placed on ice throughout the incubation period. Phagocytosis of beads was examined by flow cytometric analysis performed with a fluorescence-activated cell sorter 
(FACScan) flow cytometer (Becton Dickinson Co.). The data were analysed on monoparametric histograms [17], and expressed as percentage of positive fluorescent cells. Percentages of phagocytosing cells were calculated by subtracting percentage fluorescence-positive cells in controls $(<5 \%)$ from that of incubated cells.

\section{Chemotaxis assay}

Chemotaxis of PMNs under agarose was determined according to the method of NeLson et al. [18]. Briefly, six series of three wells, $3 \mathrm{~mm}$ in diameter and spaced $3 \mathrm{~mm}$ apart, were cut in agarose plates. Each of $10 \mu \mathrm{L}$ of formyl-methionyl-leucyl-phenylalanine (FMLP, $10^{-7} \mathrm{M}$ ) (Sigma) and Hank's balanced salt solution (HBSS) was placed in the outer and inner wells, respectively. The centre wells contained $1 \times 10^{6}$ PMNs suspended in Medium199 with culture filtrates $(0,3,5,10,20$ and $30 \%)$. The plates were incubated in a $5 \% \mathrm{CO}_{2}$ incubator for $2 \mathrm{~h}$ at $37^{\circ} \mathrm{C}$, and then fixed with ethanol and stained with Giemsa. Quantification of migration was assessed by the linear distance which the cells had moved from the margin of the centre well towards FMLP.

$\mathrm{ACF}$ was centrifuged at $5,000 \times \mathrm{g}$ at $4^{\circ} \mathrm{C}$ for $40 \mathrm{~min}$ through membrane filters (CL-LGC) (Millipore Co.) to obtain a fraction containing low molecular weight (MW) substances $(<10 \mathrm{kDa})$ (fraction I). ACF was also dialysed in a dialysis tube (MW exclusion of $10 \mathrm{kDa}$ ) against 10 volumes of Medium-199 changed hourly for $6 \mathrm{~h}$ to obtain another fraction containing high MW substances $(>10 \mathrm{kDa})$ (fraction II). Both fractions were reconstituted to the original volume with Medium-199. The antichemotactic activity of both fractions was assessed. Aliquots of ACF were treated with either trypsin (0.01 and $\left.0.1 \mathrm{mg} \cdot \mathrm{mL}^{-1}\right)$ or chicken egg white ovomacroglobulin $(\mathrm{OMG})\left(0.3\right.$ and $3.0 \mathrm{mg} \cdot \mathrm{mL}^{-1}$ ) (a generous gift from Otuka Pharmaceutical Co. Ltd) for $30 \mathrm{~min}$ at $37^{\circ} \mathrm{C}$, and their effects on the antichemotactic activity of ACF were examined.

\section{Superoxide anion assay}

Production of superoxide anion was assayed by determining superoxide dismutase (SOD)-inhibitable reduction of cytochrome C [19]. Briefly, $2.5 \times 10^{5} \mathrm{PMNs}$ were preincubated in Medium-199 containing various concentrations of culture filtrates $(0,3,6,12.5,25$ and $50 \%)$ for $60 \mathrm{~min}$ at $37^{\circ} \mathrm{C}$ and then washed with PBS. In a final volume of $500 \mu \mathrm{L}$ of HBSS containing cytochrome C $(120 \mu \mathrm{mol})($ Sigma) with or without $50 \mu \mathrm{g}$ of SOD, phorbol myristate acetate (PMA, $100 \mathrm{ng} \cdot \mathrm{mL}^{-1}$ ) (Sigma) and PMNs were incubated in a shaking water bath at $37^{\circ} \mathrm{C}$ for $30 \mathrm{~min}$. Finally, the cells were removed by centrifugation. The superoxide production was assessed in the supernatants as the difference in absorption from the control at $550 \mathrm{~nm}$ measured on a spectrophotometer (Hitachi U-3210), and amount of superoxide anion in nanomoles released per $10^{6}$ PMNs was calculated. All experiments were performed in triplicate.

\section{Assay of general proteolytic activity}

Azocoll hydrolysis was analysed by the method of CHAvir et al. [20]. Each assay tube contained $5 \mathrm{mg}$ of azocoll in $50 \mathrm{mM}$ Tris- $\mathrm{HCl}, \mathrm{pH} 8.0$, and the reaction was started by addition of ACF in a shaking water bath at $37^{\circ} \mathrm{C}$ for $30 \mathrm{~min}$. The reaction of the solution was stopped by placing tubes on ice followed by centrifugation, and absorbance of the supernatant at $520 \mathrm{~nm}$ was recorded. One unit was defined as a change of 0.001 optical density (OD) at $520 \mathrm{~nm}$ per minute at $37^{\circ} \mathrm{C}$, and $1 \mathrm{U}$ activity corresponded to $13 \mu \mathrm{g}$ protein. The protein concentration was determined by the Lowry method.

\section{Statistical analysis}

Data are presented as mean \pm SEM. Statistical comparisons between the test solutions and control were performed using Student's t-test for paired data. A p-value of less than 0.01 was considered to be significant.

\section{Results}

\section{Effect of ACF on phagocytosis by human AMs}

As shown in figure $1 \mathrm{a}$ and $\mathrm{b}$, ingestion by AMs of conidia obtained from YN strain of $A f$ was assessed in the presence or absence of ACF. In Medium-199 alone, $52 \%$ of AMs ingested conidia during the $60 \mathrm{~min}$ incubation period and the phagocytic index increased from $30 \pm 9.4(15 \mathrm{~min})$ to $126 \pm 27.0(60 \mathrm{~min})$ in a timedependent manner. However, when co-cultured with $30 \% \mathrm{ACF}$, the percentage of AMs ingesting conidia was $24 \%$ and the phagocytic index was $42 \pm 11.9$ at $60 \mathrm{~min}$. The ingestion of conidia by AMs treated with $30 \%$ ACF was significantly suppressed compared with those treated with Medium-199 alone $(\mathrm{p}<0.01)$.

The effect of ACF on nonspecific phagocytosis by AMs using latex beads was also examined as shown in table 1. The percentage of AMs associated with latex beads and the mean fluorescence intensity were both decreased significantly $(\mathrm{p}<0.01)$ with $50 \%$ ACF treatment for $60 \mathrm{~min}$. Treatment with the culture filtrates of C. albicans had no antiphagocytotic effect.

\section{Effect of ACF on chemotaxis of human PMNs}

As shown in figure 2, the migration distance of PMNs towards FMLP was reduced by ACF in a concentrationdependent manner, with $3 \%$ ACF being sufficient for the inhibitory effect $(\mathrm{p}<0.01)$. With $20 \%$ ACF, chemotaxis was within the range of random migration which was also further decreased, and PMNs treated with $30 \%$ ACF were almost immotile. The antichemotactic activity of ACF was completely abolished by autoclaving at $121^{\circ} \mathrm{C}$ for 10 min. The migration of PMNs towards ACF was 

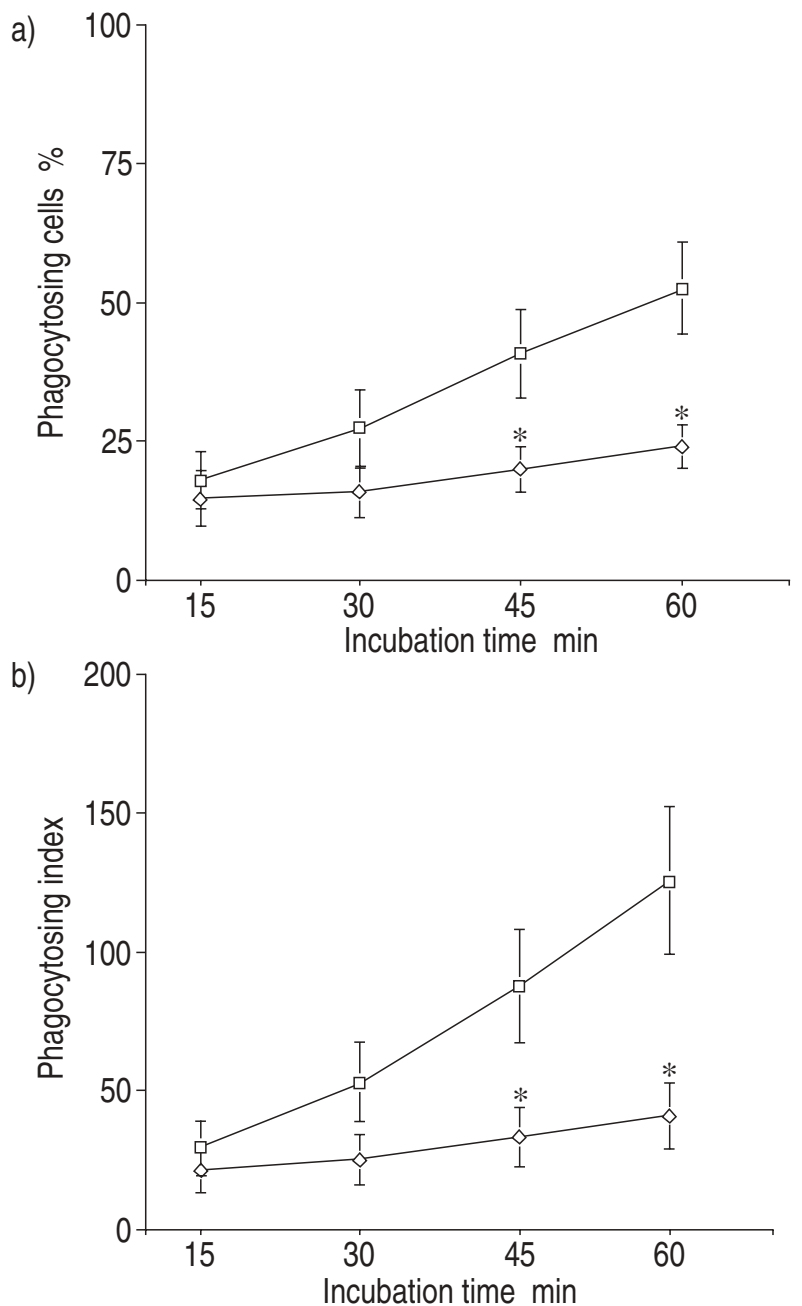

Fig. 1. - Phagocytosis of conidia by alveolar macrophages (AMs) in Medium-199 containing 30\% ACF (——-) or Medium-199 alone (control) (- —). a) Phagocytosis expressed as percentage phagocytosing cells (percentage of cells containing conidia); and b) phagocytosis expressed as phagocytic index (numbers of ingested conidia per 100 AMs) at each culture time-point $(15,30,45$ and $60 \mathrm{~min})$. The results are presented as mean \pm SEM. Each experiment was repeated four times by using AMs from different volunteers. *: $\mathrm{p}<0.01$ compared to control (Medium-199). ACF: Aspergillus fumigatus culture filtrates.

further examined and it was confirmed that ACF was not acting as a chemoattractant (data not shown).

On the other hand, culture filtrates of $C$. albicans at concentrations of $10-30 \%$ showed no significant inhibitory effect on the migration of PMNs. Moreover, as

Table 1. - Effect of culture filtrates on the association between alveolar macrophages (AMs) and latex beads

\begin{tabular}{lc}
\hline & $\begin{array}{c}\text { AMs associated with } \\
\text { latex beads \% }\end{array}$ \\
\hline Medium-199 & $57 \pm 11$ \\
$20 \%$ of ACF & $47 \pm 11$ \\
$50 \%$ of ACF & $37 \pm 11^{*}$ \\
$50 \%$ of Candida culture filtrates & $54 \pm 4$ \\
\hline
\end{tabular}

The data are presented as mean \pm SEM of four experiments. *: $\mathrm{p}<0.01$ compared with Medium-199. ACF: Aspergillus fumigatus culture filtrates.

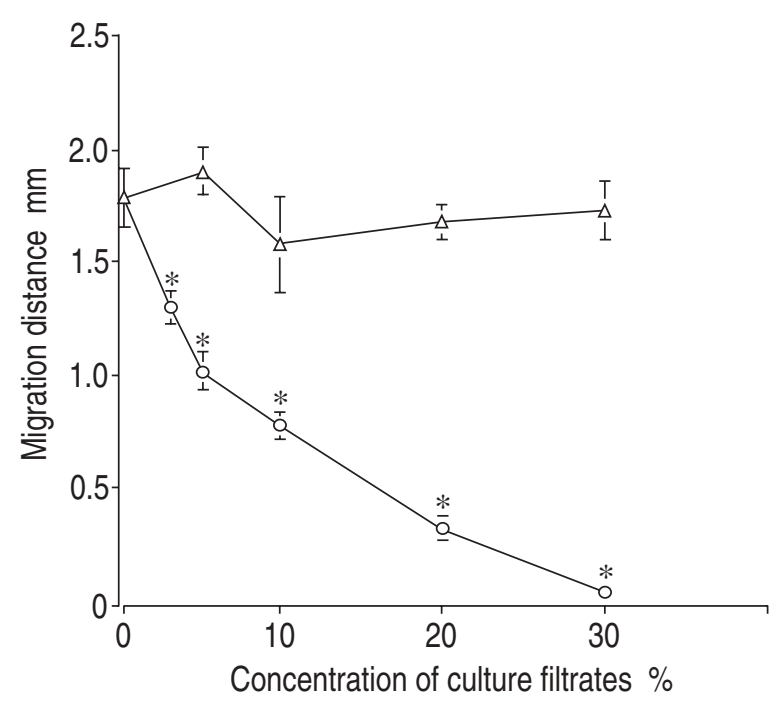

Fig. 2. - Effect of Aspergillus fumigatus culture filtrates on chemotaxis of human polymorphonuclear leucocytes (PMNs). Chemotactic activity was expressed as the linear distance $(\mathrm{mm})$ the cells had migrated towards FMLP $\left(10^{-7} \mathrm{M}\right)$. The migration distance of PMNs treated with various concentrations of culture filtrates prepared from A. fumigatus (-O-) and Candida albicans $(-\Delta-)$ were plotted. The experiments were repeated four times, and data are presented as mean \pm SEM. *: $\mathrm{p}<0.01$, compared to control (Medium-199). FMLP: formyl-methionyl-leucyl-phenylalanine.

shown in figure 3, both low MW (fraction I) and high MW (fraction II) fractions retained antichemotactic activity $(\mathrm{p}<0.01)$, although the suppressive effect of fraction I was stronger than that of fraction II throughout the range of dilution of both fractions between $10-30 \%$ in Medium-199 ( $\mathrm{p}<0.01)$. These results suggest that several factors in ACF contribute to its antichemotactic activity, and that low MW substances may be more potent than those with high MW as antichemotactic factors.

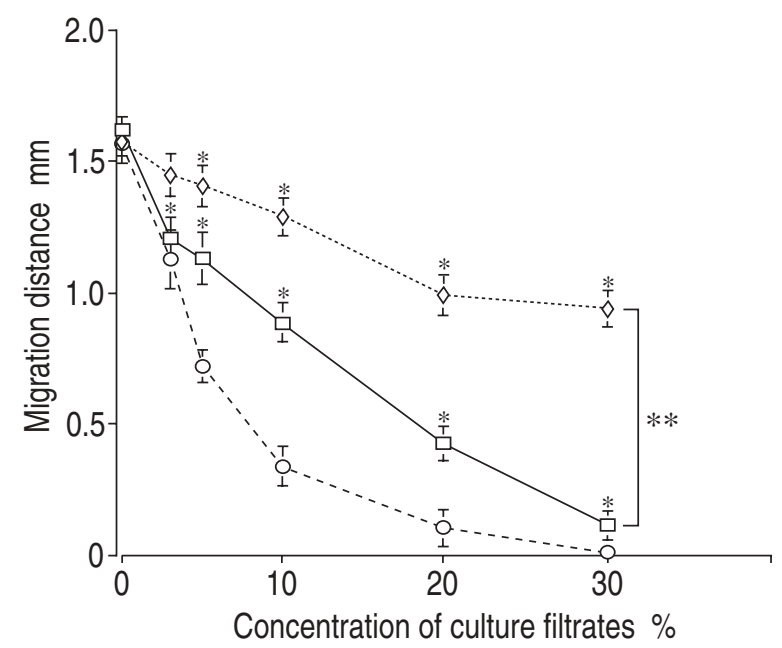

Fig. 3. - Effect of fraction I (MW $<10 \mathrm{kDa})(-\square-)$ and fraction II (MW $>10 \mathrm{kDa})(\ldots . . \cdots \ldots)$ on the migration of human polymorphonuclear leucocytes (PMNs). Although antichemotactic activity of fraction I and fraction II was reduced compared with that of original ACF ( - - O - . ), both fractions retained antichemotactic activity. The experiments were repeated three times and data are presented as mean \pm SEM. $*$ : $\mathrm{p}<0.01$, compared to control (Medium-199); **: $\mathrm{p}<0.01$, comparison between fraction I and II. MW: molecular weight; ACF: Aspergillus fumigatus culture filtrates. 


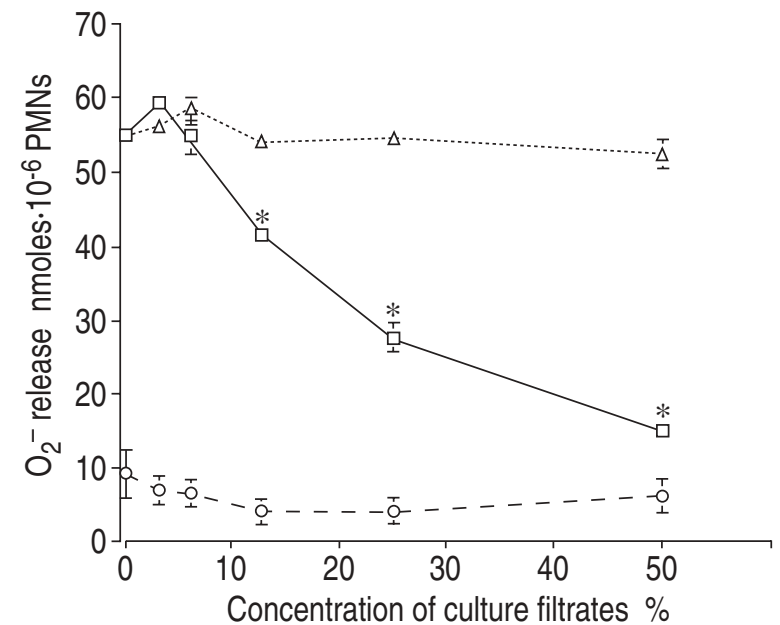

Fig. 4. - Effect of Aspergillus fumigatus culture filtrates (ACF) on superoxide anion release from human polymorphonuclear leucocytes (PMNs). Superoxide anion release (expressed as nmoles $\cdot 10^{-6} \mathrm{PMNs} \cdot 30$ min) from human PMNs treated with various concentrations of culture filtrate prepared from A. fumigatus $(\square, \bigcirc)$ and Candida albicans $(\Delta)$ were plotted. Spontaneous release of $\mathrm{O}_{2}^{-}$, shown by dashed line ( - - O - - ), was not affected by ACF treatment. PMA (100 ng.mL $\left.\mathrm{m}^{-1}\right)$ stimulated $\mathrm{O}_{2}^{-}$release was suppressed by ACF treatment, as shown by a continuous line ( $-\square-$ ), without loss of cellular viability. PMAstimulated $\mathrm{O}_{2}^{-}$release in the culture filtrates of $C$. albicans $(\cdots \cdots \Delta \cdots \cdot)$ remained unchanged. The experiments were repeated 10 times, the data are presented as mean \pm SEM. *: $\mathrm{p}<0.01$, compared to control (Medium-199). PMA: phorbol myristate acetate.

\section{Effect of ACF on superoxide anion release from PMNs}

Without stimulation, spontaneous $\mathrm{O}_{2}^{-}$release was affected neither by $A$. fumigatus nor by $C$. albicans culture filtrates throughout the $60 \mathrm{~min}$ incubation period. When PMA (100 $\mathrm{ng} \cdot \mathrm{mL}^{-1}$ ) was used as the stimulant, a shortterm exposure within $45 \mathrm{~min}$ had no significant effect on superoxide generation by PMNs (data not shown). However, as shown in figure 4, after treatment for 60 min in $12.5-50 \%$ ACF, the superoxide anion release stimulated with PMA was significantly reduced in a concentration-dependent manner compared to that in Medium-199 (53.8 \pm 1.40 nmoles $\cdot 10^{6}$ AM 30 min in medium alone, $41.6 \pm 1.40$ in $12.5 \% \mathrm{ACF}$ and $14.5 \pm 1.24$ in $50 \% \mathrm{ACF})$. ACF at concentrations between 3-6\% appeared to augment $\mathrm{O}_{2}^{-}$production slightly, but not significantly. ACF had the same effect on $\mathrm{O}_{2}^{-}$release when PMA was replaced by opsonized zymosan as the stimulant (data not shown). Since ACF itself did not influence the $\mathrm{O}_{2}^{-}$release of PMNs (data not shown), these results were not due to exhaustion of PMNs by ACF. In contrast, PMNs treated with 3-50\% culture filtrates of $C$. albicans for $60 \mathrm{~min}$ showed no significant changes in $\mathrm{O}_{2}^{-}$release in response to PMA or opsonized zymosan.

\section{Effects of trypsin and $O M G$ on biological activity of $A C F$}

As shown in figure 5, the antichemotactic effect of ACF was reduced by $56 \%$ in the presence of 0.1 $\mathrm{mg} \cdot \mathrm{mL}^{-1}$ trypsin $(\mathrm{p}<0.01)$, or by $29.5 \%$ in the presence of $3 \mathrm{mg} \cdot \mathrm{mL}^{-1} \mathrm{OMG}(\mathrm{p}<0.05)$. At these concentrations,

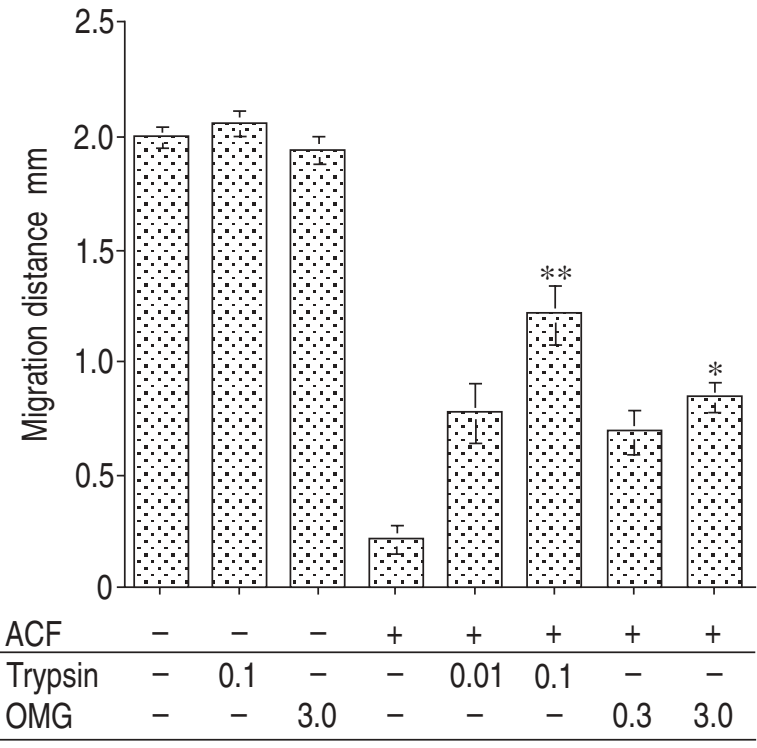

Fig. 5. - Partial inactivation of antichemotactic activity of Aspergillus fumigatus culture filtrates (ACF) by trypsin or ovomacroglobulin (OMG). ACF were pretreated with trypsin $\left(0.01\right.$ and $\left.0.1 \mathrm{mg} \cdot \mathrm{mL}^{-1}\right)$ or OMG $\left(0.3\right.$ and $\left.3.0 \mathrm{mg} \cdot \mathrm{mL}^{-1}\right)$ for $30 \mathrm{~min}$ at $37^{\circ} \mathrm{C}$. Chemotactic activity of polymorphonuclear leucocytes (PMNs) was not affected either by trypsin or OMG, compared with control (Medium-199). Data are presented as mean \pm SEM of three separate experiments. *: $\mathrm{p}<0.05$, compared with ACF; **: p<0.01, compared with ACF.

neither trypsin nor OMG per se had any effect on PMN chemotaxis. OMG was reported to be a broad-spectrum protease inhibitor and to suppress the activities of the extracellular proteases produced by Serratia marcescens and Pseudomonas aeruginosa [21]. Proteinase activity with azocoll as the substrates of the untreated ACF was $1 \mathrm{U} \cdot \mathrm{mL}^{-1}$. These results suggest that protein and proteinase account, at least partially, for the antichemotactic activity of ACF.

\section{Comparison of the biological activities of ACF and gliotoxin}

The biological activity of ACF was compared with that of gliotoxin, a secondary metabolite of $A f$, which was reported by EICHNER and co-workers $[10,11]$ to inhibit rodent macrophage functions. It was also confirmed that gliotoxin (Sigma) inhibited chemotaxis of human PMNs at 0.1 and $0.3 \mu \mathrm{g} \cdot \mathrm{mL}^{-1}$, and that the former concentration of gliotoxin had antichemotactic activity equivalent

Table 2. - Effects of gliotoxin on chemotaxis and superoxide anion release of human polymorphonuclear leucocytes (PMNs)

\begin{tabular}{lcc}
\hline $\begin{array}{l}\text { Gliotoxin } \\
\mu \mathrm{g} \cdot \mathrm{mL}^{-1}\end{array}$ & $\begin{array}{c}\text { Migration distance } \\
\mathrm{mm}\end{array}$ & $\begin{array}{c}\mathrm{O}_{2}^{-} \text {release } \\
\text { nmoles } 10^{-6} \mathrm{PMNs}\end{array}$ \\
\hline 0 & $2.05 \pm 0.05$ & $58.1 \pm 0.69$ \\
0.1 & $0.73 \pm 0.12^{*}$ & $64.2 \pm 0.98$ \\
0.3 & $0.27 \pm 0.03^{*}$ & $52.5 \pm 1.49^{*}$ \\
1.0 & $\mathrm{ND}$ & $33.3 \pm 1.55^{*}$ \\
\hline
\end{tabular}

Values are presented as mean \pm SEM. ND: not determined. *: $\mathrm{p}<0.01$. 
to that of 5-10\% ACF. However, a higher concentration of gliotoxin $\left(0.3 \mu \mathrm{g} \cdot \mathrm{mL}^{-1}\right)$ was required to suppress $\mathrm{O}_{2}^{-}$release from human PMNs stimulated with PMA (table 2).

\section{Discussion}

We have demonstrated that ACF interfered with ingestion both of Aspergillus conidia and latex beads by human AMs. In addition, ACF reduced migration of human PMNs towards FMLP and suppressed superoxide anion release from PMNs stimulated with PMA, whilst spontaneous $\mathrm{O}_{2}^{-}$release was not affected. We have also confirmed that ACF obtained from four other clinical isolates of $A f$, in addition to the YN strain, had the same sort of effects on phagocytic functions (data not shown), whereas, no such inhibitory activities were observed with $C$. albicans culture filtrates.

As pulmonary aspergillosis results from inhalation and deposition of conidia, AMs are considered to form the first line of defence against the fungal spores [22]. Both in humans and in experimental animals, neutropenia and dysfunction of PMNs are the greatest risk factors for invasive aspergillosis [6, 23]. Cellular and humoral immunity are not considered to contribute to the host defence in aspergillosis [3, 4, 17, 22]. Alveolar macrophages and neutrophils appear to be the primary components of the host defence network, and play essential roles in eradication of the fungus $[3,4,6,24]$. Phagocytic functions, such as ingestion, migration and release of reactive oxygen intermediates, which appear to be responsible for the killing activity, are important host defence mechanisms against $A f$. Evasion of these functions may be the principal tools by which Af persist in the sites of colonization and proliferate further.

AMs killed conidia more efficiently than peritoneal macrophages of rabbits, which suggests that the anatomical site of macrophages influences their activities against Af [3]. Some groups have analysed the interactions between Aspergillus and phagocytes, and have shown that several fungal metabolites interfere with phagocytic function. However, these previous studies mainly used phagocytes obtained from experimental animals [7-11, 25]. Therefore, corresponding experiments on human AMs and PMNs are necessary before any extrapolation can be made to human pulmonary aspergillosis. In the present study, we examined the interactions between human phagocytes and ACF in vitro. Our results suggest that $A$. fumigatus releases factors which impair the functions of human AMs and PMNs, and consequently escapes the local host defences. It is meaningful that ACF of the YN strain itself suppressed the ingestion of conidia derived from this strain by AMs.

WASHBURN et al. [25] reported that $A f$ produced a soluble extracellular inhibitor of the alternative complement pathway, and showed that this material interfered with C3b-dependent phagocytosis and killing. SCHAFFNER [22] reported that resident AMs ingested conidia in the absence of antibodies, thus permitting phagocytosis of conidia by AMs in an environment where serum components were not readily available. Since the conidia used in our study were not opsonized, it seems that the inhibitory effect of ACF on phagocytosis was not due to the mechanism reported by WASHBURN et al. [25].

EICHNER and co-workers $[10,11]$ reported that a 3 day culture of $A f$ generated metabolites with antiphagocytic activity, namely inhibition of phagocytosis of carbon particles by mouse peritoneal exudate cells, and adherence to plastic surfaces by rodent peritoneal and alveolar macrophages, and also inhibited the basal rate of $\mathrm{H}_{2} \mathrm{O}_{2}$ production by human PMNs. These activities were stable against digestion by trypsin and protease, and the factors responsible had a MW less than $0.5 \mathrm{kDa}$. They identified one of the biologically active components as gliotoxin $[10,11]$. Gliotoxin has been isolated from cultures of $A f$ as well as other fungi, including Gliocladium fimbriatum, Penicillium obscurum, and Trichoderma lignorum, and belongs to the epipolythiodioxopiperazine group. Most members of this group are known to possess some biological activities [26].

We also confirmed that gliotoxin interfered with migration of PMNs and PMA-induced $\mathrm{O}_{2}^{-}$release from PMNs. However, at least some of the antichemotactic components of ACF consisted of protein and were associated with the high MW fraction $(>10 \mathrm{kDa})$ as well as the low MW fraction.

ROBERTSON and co-workers [7-9] reported that $A f$ spore diffusates, obtained from $3 \mathrm{~h}$ culture supernatants, reduced the migration of human PMNs, the spreading of mouse peritoneal exudate cells, and the spontaneous $\mathrm{O}_{2}^{-}$ release from mouse peritoneal exudate cells or BAL cells of Corynebacterium parvarum-treated rats. The spore diffusates were obtained after only $3 \mathrm{~h}$ of culture, whilst gliotoxin and ACF used in the present study were obtained after 3-5 days of culture, when mycelial growth would be abundant. In human tissues, $A f$ grows almost exclusively in the mycelial form. Consequently, to progress from simple colonization of $A f$ to semi-invasive or invasive pulmonary aspergillosis, the latter factors are considered to be more important than the spore diffusates obtained after short-term culture.

Several researchers have reported the isolation and characterization of extracellular elastinolytic proteases from $A f$. These enzymes appeared to be serine proteases [27] or metalloproteases [28]. KothARy et al. [29] reported that elastase activity was correlated with virulence for mice, and RHODES et al. [30] reported that all isolates involved in human invasive aspergillosis displayed elastinolytic activity in vitro. Although they suggested that digestive exoenzymes degrade the structural barriers in the host, they did not comment on the relationships between elastase and phagocytic functions.

The results of the present study suggest that part of the antichemotactic activity is associated with protease. Recently, HoLDEN and co-workers [31] established a protease gene-disrupted mutant of $A f$, and reported no differences between mutant and wild-type strain in terms of mortality or histological features in murine models for aspergillosis. We have tested culture filtrates of the mutant strain (a kind gift from D.W. Holden), which 
lacked extracellular proteolytic activity. We confirmed that culture filtrates of the mutant strain exhibited antichemotactic activity and suppressed PMA-induced $\mathrm{O}_{2}^{-}$ release, although the activities were lower than that of the culture filtrates of the wild-type strain (data not shown).

Thus, we conclude that several factors participate in the antiphagocytic activity of ACF. Such candidates may be low MW substances, probably including gliotoxin, and high MW substances, such as proteases and other unidentified substances. It will be an important future work to identify the substances in ACF and to clarify the mechanisms of antiphagocytic effects.

We have previously shown that most ACFs of clinically isolated $A f$, including the $\mathrm{YN}$ strain, slow ciliary beat frequency associated with marked disruption of human respiratory ciliated epithelium, probably leading to impairment of the mucociliary clearance, which is the most important first line of defence in the airways [12]. AMitani et al. [13] isolated the cilioinhibitory substances from $\mathrm{ACF}$, and identified one of the potent substances as gliotoxin. Consequently, Af must be capable of circumventing the elaborate defence network both in the airways and the lungs in order to persist and proliferate there.

The final role of PMNs in host defence against Aspergillus is in hyphal damage. Additional studies to determine whether ACF suppresses the hyphal damage by PMNs are currently in progress. The demonstration of the antiphagocytic substances in human tissues infected by $A f$ will also be required to clarify the pathogenetic mechanisms of colonization and invasion of $A f$ in the lungs.

Acknowledgements: The authors wish to thank D.W. Holden, the Department of Infectious Diseases and Bacteriology, the Royal Postgraduate Medical School, London, for providing the mutant strains of A. fumigatus. The authors are grateful to T. Homma, T. Ueda and K. Kataoka for their kind assistance.

\section{References}

1. Binder RE, Faling LJ, Pugatch RD, Mahasean CM, Snider GL. Chronic necrotizing pulmonary aspergillosis: discrete clinical entity. Medicine 1982; 61: 109124.

2. Schaffner A, Douglas H, Braude A. Selective protection against conidia by mononuclear and against mycelia by polymorphonuclear phagocytes in resistance to Aspergillus. J Clin Invest 1982; 69: 617-631.

3. Schaffner A, Douglas H, Braude A, Davis CE. Killing of Aspergillus spores depends on the anatomical source of the macrophage. Infect Immun 1983; 42: 1109-1115.

4. Levitz SM, Diamond RD. Mechanisms of resistance of Aspergillus fumigatus conidia to killing by neutrophils in vitro. J Infect Dis 1985; 152: 33-42.

5. Diamond RD, Clark RA. Damage to Aspergillus fumigatus and Rhizopus oryzae hyphae by oxidative and nonoxidative microbicidal products of human neutrophils in vitro. Infect Immun 1982; 38: 487-495.
6. Rex JH, Bennett JE, Gallin JI, Malech HL, Melnick DA. Normal and deficient neutrophils can co-operate to damage Aspergillus fumigatus hyphae. J Infect Dis 1989; 162: 523-528.

7. Robertson MD, Seaton A, Milne LJR, Raeburn JA. Resistance of spores of Aspergillus fumigatus to ingestion by phagocytic cells. Thorax 1987; 42: 466-472.

8. Robertson MD, Seaton A, Milne LJR, Raeburn JA. Suppression of host defences by Aspergillus fumigatus. Thorax 1987; 42: 19-25.

9. Robertson MD, Seaton A, Raeburn JA, Milne L Jr. Inhibition of phagocyte migration and spreading by diffusates of Aspergillus fumigatus. J Med Ver Mycology 1987; 25: 389-396.

10. Mullbacher A, Waring P, Eichner RD. Identification of an agent in cultures of Aspergillus fumigatus displaying antiphagocytic and immunomodulating activity in vitro. J Gen Microbiol 1985; 131: 1251-1258.

11. Eichner RD, Salami MAL, Wood PR, Mullbacher A. The effect of gliotoxin upon macrophage function. Int J Immunopharmacol 1986; 8: 789-797.

12. Amitani R, Murayama $\mathrm{T}$, Nawada R, et al. Aspergillus culture filtrates and sputum sols from patients with pulmonary aspergillosis cause damage to human respiratory ciliated epithelium. Eur Respir J 1995; 8: 1681-1687.

13. Amitani R, Taylor G, Elezis E-N, et al. Purification and characterization of factors produced by Aspergillus fumigatus which affect human ciliated respiratory epithelium. Infect Immun 1995; 63: 3266-3271.

14. Robinson BWS, Venaille TJV, Mendis AHW, McAleer R. Allergens as proteases: an Aspergillus fumigatus proteinase directly induces human epithelial cell detachment. J Allergy Clin Immunol 1990; 85: 726-731.

15. Anderson R, Picht J, Lohr PG. Primary culture of human blood-born macrophages grown on hydrophobic Teflon membranes. J Immunol Meth 1983; 56: 295-304.

16. Hed J. Methods for distinguishing ingested from adhering particles. Methods Enzymol 1986; 6: 198-204.

17. Repentigny L, Petitbois S, Boushira M, et al. Acquired immunity in experimental murine aspergillosis is mediated by macrophages. Infect Immun 1993; 61: 37913802 .

18. Nelson R, Que PG, Simmons RL. Chemotaxis under agarose: a new and simple method for measuring chemotaxis and spontaneous migration of human polymorphonuclear leukocytes and monocytes. J Immunol 1975; 115: 1650-1656.

19. Johnston RB Jr, Godzik CA, Cohn ZA. Increased superoxide anion production by immunologically-activated and chemically-elicited macrophages. J Exp Med 1978; 148: 115 .

20. Chavir R Jr, Burnett TJ, Hageman JH. Assaying proteinases with azocoll. Anal Biochem 1984; 136: 446450 .

21. Miyagawa S, Matsumoto K, Kamata R, Okamura R, Maeda H. Spreading of Serratia marcescens in experimental leratitis and growth suppression by chicken egg white ovomacroglobulin. Jpn J Ophthamol 1991; 35: 402-410.

22. Schaffner A. Macrophage-Aspergillus interactions. Immunol Ser 1994; 60: 545-552.

23. Denning DW, Follansbee SE, Scolad M, Norris SN, Edelstein H, Stevens DA. Pulmonary aspergillosis in the acquired immunodeficiency syndrome. $N$ Engl $\mathrm{J} \mathrm{Med}$ 1991; 324: 654-662.

24. Levitz SM, Selsted ME, Ganz T, Leherer RI, Diamond RD. In vitro killing of spores and hyphae of Aspergillus 
fumigatus and Rhizopus oryzae by rabbit neutrophil cationic peptides and bronchoalveolar macrophages. J Infect Dis 1986; 154: 483-489.

25. Washburn RG, Hammer CH, Bennett JE. Inhibition of complement by culture supernatants of Aspergillus fumigatus. J Infect Dis 1986; 154: 944-951.

26. Betina V. Gliotoxins. In: Betina V, eds. Bioactive Molecules. Vol. 9. Mycotoxins: chemicals, biological and environmental aspects. Amsterdam, Elsevier, 1989; pp. 388-392.

27. Kolattukuy PE, Lee JD, Rogers LM, et al. Evidence for involvement of an elastinolytic serine protease in aspergillosis. Infect Immun 1993; 61: 2357-2368.

28. Markaryan A, Morozova I, Yu H, Kolattukudy PE. Purification and characterization of an elastinolytic metalloprotease from Aspergillus fumigatus and immunoelectron microscopic evidence of secretion of this enzyme by the fungus invading the murine lung. Infect Immun 1994; 62: 2149-2157.

29. Kothary MH, Chase T Jr, Macmillan JD. Correlation of elastase production by some strains of Aspergillus fumigatus with ability to cause pulmonary invasive aspergillosis in mice. Infect Immun 1984; 43: 320325.

30. Rhodes JC, Bode RB, McCuan-Kirsch CM. Elastase production in clinical isolates of Aspergillus. Diag Microbiol Infect Dis 1988; 10: 165-170.

31. Tang C, Cohen J, Krausz T, Van Noorden S, Holden DW. The alkaline protease of Aspergillus fumigatus is not a virulence determinant in two murine models of invasive pulmonary aspergillosis. Infect Immun 1993; 61: $1650-1656$. 